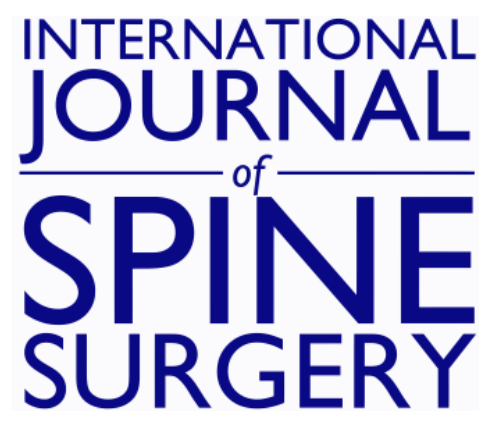

\title{
Cell and Tissue Response to Polyethylene Terephthalate Mesh Containing Bone Allograft in Vitro and in Vivo
}

D. Joshua Cohen, Lisa Ferrara, Marcus B. Stone, Zvi Schwartz and Barbara D. Boyan

Int J Spine Surg 2020, 14 (s3) S121-S132

doi: https://doi.org/10.14444/7135

http://ijssurgery.com/content/14/s3/S121

This information is current as of April 26, 2023.

Email Alerts Receive free email-alerts when new articles cite this article. Sign up at:

http://ijssurgery.com/alerts

The International Journal of Spine Surgery

2397 Waterbury Circle, Suite 1,

Aurora, IL 60504, Phone: +1-630-375-1432 


\title{
Cell and Tissue Response to Polyethylene Terephthalate Mesh Containing Bone Allograft in Vitro and in Vivo
}

\author{
D. JOSHUA COHEN, MD, ${ }^{1}$ LISA FERRARA, PHD,${ }^{2}$ MARCUS B. STONE, PHD, ${ }^{3}$ ZVI SCHWARTZ, DMD, PHD,${ }^{1,4}$ \\ BARBARA D. BOYAN, PHD ${ }^{1,5}$ \\ ${ }^{1}$ Department of Biomedical Engineering, Virginia Commonwealth University, Richmond, Virginia, ${ }^{2}$ OrthoKinetic Technologies, Southport, North Carolina, ${ }^{3}$ Spine \\ Institute of Louisiana, Shreveport, Louisiana, ${ }^{4}$ Department of Periodontics, University of Texas Health Science Center at San Antonio, San Antonio, Texas, \\ ${ }^{5}$ Wallace H. Coulter Department of Biomedical Engineering, Georgia Institute of Technology, Atlanta, Georgia
}

\begin{abstract}
Background: Extended polyethylene terephthalate mesh (PET, Dacron) can provide containment of compressed particulate allograft and autograft. This study assessed if PET mesh would interfere with osteoprogenitor cell migration from vertebral plates through particulate graft, and its effect on osteoblast differentiation or the quality of bone forming within fusing vertebra during vertebral interbody fusion.

Methods: The impact of PET mesh on the biological response of normal human osteoblasts (NHOst cells) and bone marrow stromal cells (MSCs) to particulate bone graft was examined in vitro. Cells were cultured on rat bone particles +/- mesh; proliferation and osteoblast differentiation were assessed. The interface between the vertebral endplate, PET mesh, and newly formed bone within consolidated allograft contained by mesh was examined in a sheep model via microradiographs, histology, and mechanical testing.

Results: Growth on bone particles stimulated proliferation and early differentiation of NHOst cells and MSCs, but delayed terminal differentiation. This was not negatively impacted by mesh. New bone formation in vivo was not prevented by use of a PET mesh graft containment device. Fusion was improved in sites containing allograft/ demineralized bone matrix (DBM) versus autograft and was further enhanced when stabilized using pedicle screws. Only sites treated with allograft/DBM+screws exhibited greater percent bone ingrowth versus discectomy or autograft. These results were mirrored biomechanically.

Conclusions: PET mesh does not negatively impact cell attachment to particulate bone graft, proliferation, or initial osteoblast differentiation. The results demonstrated that bone growth occurs from vertebral endplates into graft material within the PET mesh. This was enhanced by stabilization with pedicle screws leading to greater bone ingrowth and biomechanical stability across the fusion site.

Clinical Relevance: The use of extended PET mesh allows containment of bone graft material during vertebral interbody fusion without inhibiting migration of osteoprogenitor cells from vertebral end plates in order to achieve fusion.

Level of Evidence: 5.
\end{abstract}

Special Issue

Keywords: Dacron mesh, bone marrow stromal cells, MSCs, allograft

\section{INTRODUCTION}

During incremental bone formation typical of bone healing, bone marrow stromal cells (MSCs) migrate from existing bone to sites of injury. ${ }^{1}$ When bony fracture ends are in close apposition, the cells migrate across the fibrin clot and haematoma, differentiate into bone forming osteoblasts and synthesize primary bone, which is ultimately remodeled into lamellar bone. ${ }^{2}$ Osteoblast progenitor cells also migrate from the existing bone and contribute to bone regeneration. When the fracture ends are not apposed, as is seen in critical size defects and during vertebral interbody fusion, it is necessary to provide an osteoconductive surface to enable MSC and osteoprogenitor cell migration. $^{3}$

A variety of approaches have been used clinically to facilitate the fusion of vertebra, including metal and polymer cages to restore the interbody space. ${ }^{3-8}$ These devices generally consist of a surface that interfaces with the vertebral endplates and one or more channels that provide space for bony ingrowth. Frequently, channels are packed with bone graft or bone graft substitutes to facilitate MSC and osteoprogenitor cell migration and in some instances, osteogenic additives like bone marrow aspirate 
and osteoinductive agents like demineralized bone matrix (DBM) and bone morphogenetic protein 2 (BMP2) are added to stimulate bone formation. ${ }^{9-11}$ Biological materials such as segments of cortical bone allografts are also used to retain interbody space and contain any additives. ${ }^{12}$ In general, the cortical bone is not resorbed or is resorbed only slowly, ${ }^{13}$ so the effect on fusion is similar to that seen when using a simple metal or polymer cage.

A third option is the use of compressed particulate graft material consisting of allograft and autograft, which provides physical stability as well as osteogenic signals and a biologically attractive surface for cell migration, ${ }^{14}$ but lacks a containment mechanism to prevent loss of particulate from the interbody space. Extended polyethylene terephthalate mesh (PET, Dacron) can overcome this by providing the containment necessary. ${ }^{15,16}$ However, it is not known if such an approach would interfere with the migration of osteoprogenitor cells from the vertebral plates through the particulate graft, with the ability of these cells to differentiate into osteoblasts, or with the quality of bone forming within the fusing vertebra.

In order to address these questions, we used 2 interrelated approaches. Whether PET mesh would impact the biological response of MSCs and osteoprogenitor cells to allograft bone particles was assessed in vitro. The interface between the vertebral endplate, PET mesh, and newly formed bone within the consolidated allograft contained with the mesh was examined using an interbody spine fusion model in sheep and assessed qualitatively (histology) and quantitatively (microradiographs, mechanical testing).

\section{METHODS}

\section{In Vitro Culture}

Human MSCs (male donor) and normal human osteoblasts (NHOst cells, male donor), as well as MSC growth media (MSCGM) and osteogenic media (OM), were purchased from Lonza (Basel, Switzerland). Cells were cultured on bone particles prepared from long bones harvested from Sprague Dawley rats. After stripping the periosteum and bone marrow, bones were washed with Dulbecco's modified Eagle medium (DMEM) containing 150 $\mathrm{U} / \mathrm{mL}$ penicillin/streptomycin and then ground in a milling machine.

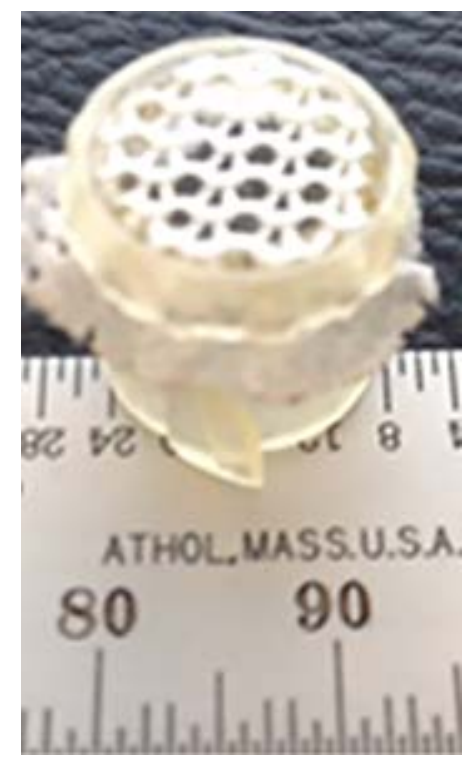

Figure 1. CellCrown inserts (Sigma-Aldrich) with sterile polyethylene terephthalate $(\mathrm{PET})$ meshes used in all in vitro experiments.

We took advantage of a method that we have used successfully to examine cell responses to biomaterials. ${ }^{17-19}$ Cell phenotype was assessed 24 hours postconfluence when differentiation was initiating (DNA content, alkaline phosphatase specific activity). We also measured production of factors associated with osteogenesis, including BMPs 2 and 4 and vascular endothelial growth factor-A (VEGF), which stimulates angiogenesis. At later states of osteoblast differentiation, cells produce osteocalcin, which limits the size of hydroxyapatite crystals and provides a chemotactic factor for osteoclasts, and osteoprotegerin (OPG), which is a decoy receptor for RANKL, thereby reducing osteoclast activity and promoting net new bone formation.

To determine if the presence of the mesh was a factor in the cell response to the bone graft, we developed an insert to overlay the bone graft. CellCrown inserts (Sigma-Aldrich, St. Louis, MO) with sterile PET meshes were supplied by Spineology (Figure 1). MSCs and NHOst cells were cultured on bone particles $(250 \mathrm{mg} /$ well $)$ in 24-well plates (6 cultures/variable); cells on tissue culture polystyrene (TCPS) were used as controls. One half of the cultures had inserts with PET mesh while the other half had empty inserts. Thus, 4 treatment groups were assessed: TCPS plus empty insert; TCPS plus insert with mesh; particulate plus empty insert; and particulate plus insert with mesh. Cells were seeded at 20,000 cells/well. Cell attachment to the mesh after 24 hours was assessed by scanning 
Table 1. Design of normal human osteoblast experiment.

\begin{tabular}{lccc}
\hline Group Number & Bone Graph & Insert & Mesh \\
\hline 1 & No & Yes & No \\
2 & No & Yes & No \\
3 & Yes & Yes & Yes \\
4 & Yes & Yes & Yes \\
\hline
\end{tabular}

electron microscopy (SEM) of the mesh in 3 separate locations.

Fresh media were added to NHOst cells at 24 hours and then at 48-hour intervals. Cells achieved confluence at 8 days and were harvested on day 9 (Table 1).

MSCs were plated in MSCGM. Forty-eight hours after plating, one-half of the cultures were switched to OM, whereas the other half remained in MSCGM. Cells were cultured for 6 days in MSCGM or OM, with media changes every 48 hours. On day 8 , fresh media were added, and cells were harvested on day 9 (Table 2).

At harvest, the conditioned media were collected. Cell layers were washed 2 times with phosphate buffered saline (PBS), lysed with $0.05 \%$ Triton X100 and homogenized by sonication. Alkaline phosphatase specific activity was measured in cell layer lysates and normalized to protein content. Osteocalcin, OPG, VEGF, BMP2, and BMP4 in the conditioned media were measured using enzyme linked immunosorbent assays (ELISAs; R\&D Systems, Minneapolis, MN) and normalized to DNA content in cell layer lysates.

\section{In Vivo Study}

All animal procedures commenced following approval by the Thomas D. Morris, Inc, Institutional Animal Care and Use Committee. The experimental design required 4 groups of 6 sites per treatment regimen. To achieve this, a total of 12 skeletally mature Western Crossbred ewes were used to evaluate the use and effectiveness of the PET mesh graft containment device (OptiMesh, Spine-

Table 2. Design of human mesenchymal stromal cell experiment.

\begin{tabular}{lcccl}
\hline Group Number & Bone Graft & Insert & Mesh & Culture Media \\
\hline 1 & No & Yes & No & Growth media \\
2 & No & Yes & No & Growth media \\
3 & Yes & Yes & Yes & Growth media \\
4 & Yes & Yes & Yes & Growth media \\
5 & No & Yes & No & Osteogenic media \\
6 & No & Yes & No & Osteogenic media \\
7 & Yes & Yes & Yes & Osteogenic media \\
8 & Yes & Yes & Yes & Osteogenic media \\
\hline
\end{tabular}

Table 3. Design of Ovine in vivo experiment.

\begin{tabular}{lc}
\hline Treatment Groups & $\begin{array}{c}\text { Schedule of Evaluations } \\
\text { (24 Weeks Postoperatively) }\end{array}$ \\
\hline Discectomy & Group $1(\mathrm{n}=6)$ \\
Autograft & Group 2 $(\mathrm{n}=6)$ \\
Allograft/DBM & Group 3 $(\mathrm{n}=6)$ \\
Allograft/DBM screws & Group 4 $(\mathrm{n}=6)$ \\
\hline
\end{tabular}

Note, two intervertebral levels were reconstructed in each animal and groups 2 through 4 used PET dacron mesh for containment.

Abbreviation: DBM, demineralized bone matrix

ology, Inc, St. Paul, MN) for stabilization and containment of various bone graft materials used for interbody spinal arthrodesis. The surgical procedure consisted of a retroperitoneal and posterolateral exposure of the L2-L3 and L4-L5 operative levels, followed by application of 1 of 4 randomized implant treatments $(\mathrm{N}=6$ sites per treatment group; thus, 3 animals were treated at L2L3 and 3 animals were treated at L4-L5). ${ }^{20-22}$ All experimental animals were euthanized at 6 months postoperatively. Four additional animals used as allograft donors were not included in the postoperative survival period.

Treatment groups (Table 3) were as follows: (1) Discectomy - no implant; (2) Autograft (morselized iliac crest prepared at time of surgery); (3) Allograft/ DBM; and (4) Allograft/DBM with pedicle screws. The allograft/DBM implants were $50 \%$ sheep cortical bone chips ( $<2 \mathrm{~mm}$ diameter) and 30\% sheep DBM powder plus $20 \%$ gelatin.

\section{Retroperitoneal Surgical Technique}

All 12 animals received surgery via the retroperitoneal technique. Following right lateral decubitus positioning and aseptic preparation, surgical exposure consisted of a $10-$ to $15-\mathrm{cm}$ incision beginning at the iliac crest and extending along the palpable borders of the lumbar transverse processes. Detachment of the external abdominal oblique from the transverse processes was performed, followed by soft tissue dissection to permit exposure of the psoas muscle. Intraoperative radiographic confirmation of the lumbar region verified the operative treatment levels. A partial discectomy was performed at each arthrodesis level through a portal in the anterolateral annulus. Disc spaces were then reamed, prepared, and either left untreated (Group 1) or were implanted with the appropriate device strictly according to the manufacturer's recommendations for each treatment procedure (Groups 2-4) (Table $3)$. Cancellous bone graft was harvested from the iliac crest and was used to pack the PET mesh 
device in Group 2 so that the device was fully extended. Similarly, allograft/DBM was packed into the device, as required. For wound closure, the muscles and fascia were approximated using 1-0 Vicryl and the skin closed with 1-0 Vicryl suture.

\section{Posterolateral Exposure and Pedicle Screw Surgical Technique}

Three of the animals that were treated anteriorly with allograft/DBM were stabilized posteriorly using transpedicular screw fixation. Following prone positioning and aseptic preparation, an initial skin incision was made in the dorsal mid-line of the low back centered over the L2-S1 levels. The L2-L3 and L4-L5 operative levels were instrumented with the TSRH Spinal System (Medtronic, Memphis, TN) posterior pedicle screw and rod system using standard technique for pedicle preparation and screw placement. Only allograft/DBM was used in the instrumented animals.

\section{Euthanasia}

Animals in each group were humanely euthanized 6 months postoperatively. The spinal column of the animal was carefully removed and immediately placed in double wrapped plastic specimen bags and frozen at $-25^{\circ} \mathrm{C}$ for subsequent radiographic, biomechanical, and histologic evaluation of the operative motion segments.

\section{Biomechanical Testing}

Frozen specimens were thawed at room temperature, and surrounding soft tissue and musculature removed to obtain the operative ligamentous functional spinal units. The superior half of the proximal vertebral body and inferior half of the distal body were secured into rectangular tubing foundations using 4 four-point compression screws. All biomechanical testing was performed using an MTS 858 materials test machine configured with an OptoTrak 3020 Motion Analysis System and on-line computerized data acquisition using OptoTrak ODAU software.

The peak range of motion (ROM; degrees) of each spinal unit was quantified under axial rotation ( $\pm 4 \mathrm{Nm}$ with $-150 \mathrm{~N}$ compressive preload), flexionextension $( \pm 4 \mathrm{Nm})$, and lateral bending $( \pm 4 \mathrm{Nm})$ for nondestructive testing modes using a pure moment loading system and a loading rate of $20 \%$ full scale/second. The initial axis of rotation was centered at the junction of the posterior one-third and anterior two-thirds of the intervertebral disc during torsion and flexion-extension testing, and adhered to the mid-coronal point of the intervertebral disc for lateral bending.

For animals treated with allograft/DBM stabilized with pedicle screws (Group 4), the operative functional units were tested with pedicles screws, followed by repeat testing upon removal of the screws. However, removal of the pedicle screws for the allograft/DBM with screws group included dissection of the facet joints at the site of implantation. Each test was repeated for 4 loading and unloading cycles, with ROM calculated at the peak applied load $(\mathrm{Nm})$ acquired from the fourth loading cycle. The peak ROM for each loading mode was calculated as the sum of motions [maximum displacement (degrees)] for torsion, flexion-extension, and lateral bending occurring in the neutral and elastic zones at the fourth loading cycle.

\section{Histopathology and Histomorphometry}

Upon completion of the biomechanical analysis, interbody operative sites were sectioned in the parasagittal planes, dehydrated in $80 \%$ ethanol, stained using Villanueva Osteochrome Bone Stain, and embedded in polymethylmethacrylate. Embedded specimens were ground and polished to $100-\mu \mathrm{m}$ thickness.

Microradiographs of histologic sections were obtained using a Faxitron X-ray Unit and Konica Graphic Arts Film. Slide-mounted specimens were placed 12 inches from the beam and exposed for 2 minutes, using a technique of $25 \mathrm{kVp}$ and $3 \mathrm{~mA}$ while in direct contact with the single emulsion highresolution graphics arts film. Resulting high-resolution microradiographs were used for morphometric quantification of trabecular bone areas (percentage) within the confines of the PET mesh material (BioQuant Image Analysis System, Nashville, TN). Fusion assessment (yes, partial, or no) was determined by blinded review of the microradiographs. A successful fusion was indicated by the presence of contiguous bone between adjacent vertebral bodies, without evidence of radiographic lucence. Based on histologic microradiographs, any incidence of PET mesh collapse, tears, or graft extrusion was recorded.

Histopathologic interpretation was based on a review of undecalcified specimens in each treatment group. The description of the histology represents a culmination of readings for each group. Two para- 
A
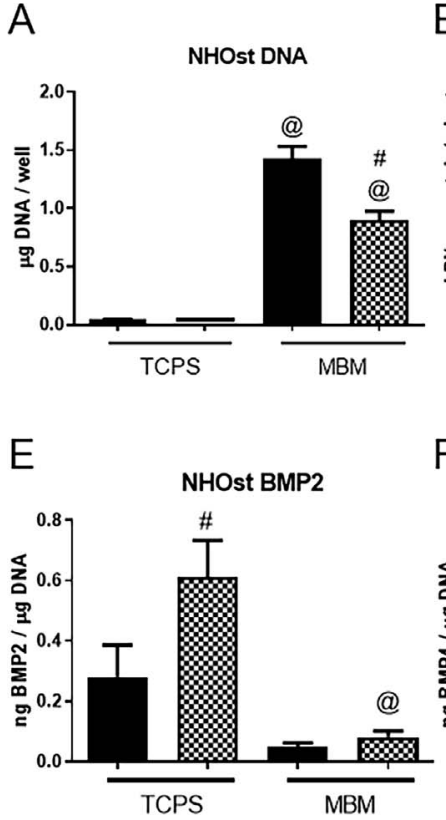
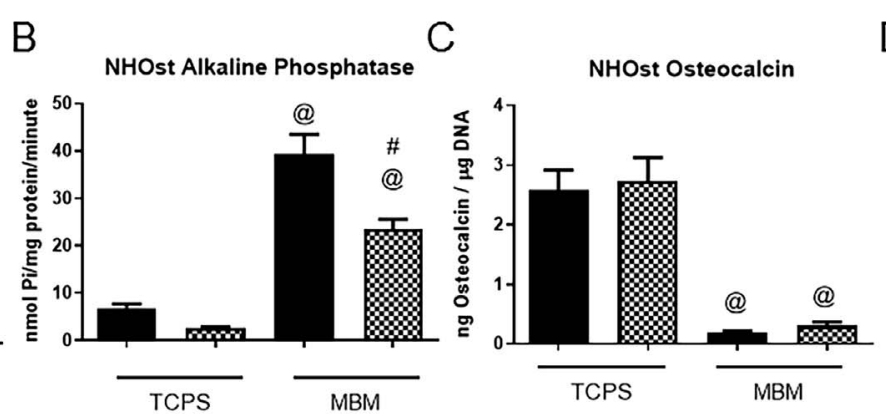

$\mathrm{F}$

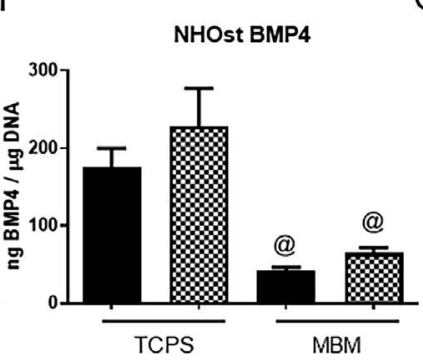

G

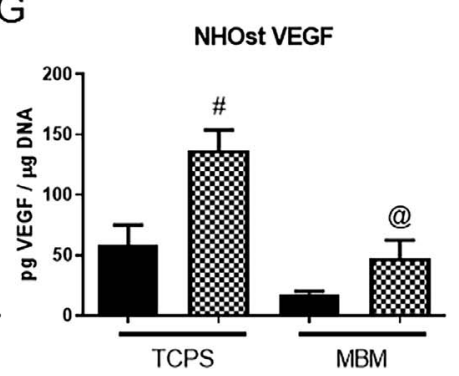

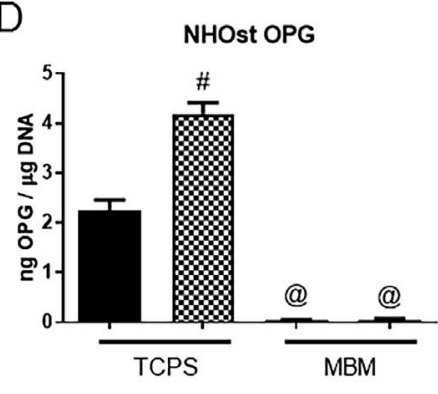

$\infty$ no Mesh

$\cos +1$

Figure 2. In vitro normal human osteoblasts (NHOst) response of cells cultured on tissue culture plastic (TCPS) or particulate bone graft (mineralized bone matrix [MBM]) in the presence or absence of polyethylene terephthalate mesh (PET). Response was examined by measuring markers that indicate the initiation of differentiation including (A) DNA; (B) alkaline phosphate specific activity; factors that indicate late stage osteoblast differentiation including (C) osteocalcin and (D) osteoprotegerin (OPG); and production of factors associated with osteogenesis, including bone morphogenetic proteins (BMPs) 2 (E) and 4 (F) and (G) vascular endothelial growth factor-A (VEGF). $P<.05$ \# versus no mesh, @ versus no MBM.

sagittal sections were reviewed for each operative level.

\section{STATISTICAL METHODS}

In vitro data are means \pm standard error of 6 independent cultures/variable. All experiments were repeated to ensure validity of observations, with results from individual experiments shown. Statistical analysis among groups was performed by 1-way analysis of variance (ANOVA), and multiple comparisons between groups were conducted with a 2-tailed Tukey correction.

For nondestructive biomechanical analysis, the peak ROM for each loading mode was calculated as the sum of motions [maximum displacement (degrees)] for torsion, flexion-extension, and lateral bending occurring in the neutral and elastic zones at the fourth loading cycle. Descriptive statistics are shown as the mean \pm 1 standard deviation and were statistically compared using ANOVA.

Histomorphometric data represent the percentage of trabecular bone within the confines of the PET Dacron mesh material. All data are shown as mean \pm 1 standard deviation and were statistically compared using ANOVA with a 2-tailed Tukey correction.
For all analyses, a $P$ value less than .05 was considered statistically significant. All statistical analysis was performed with GraphPad Prism version 5.04 .

\section{RESULTS}

\section{Cell Attachment to Mesh}

SEMs of the mesh at 24 hours postplating showed only minimal attachment of NHOst cells or MSCs and no cells were found at 48 hours. At 7 days, cells were present on the mesh, but only at very low numbers and distribution was random.

\section{NHOst Response}

Cell number (DNA) was highest on bone particles (Figure 2a), which may have reflected not only growth in the number of plated cells but also cells resident in the particulate that had migrated onto the chip surface. Alkaline phosphatase activity was highest on the bone particles compared with TCPS (Figure 2b), whereas osteocalcin production was lowest (Figure 2c), indicating an early stage of osteoblastic differentiation. Cells cultured on bone particles with mesh inserts present had lower DNA and alkaline phosphatase than when no mesh was present. 
A

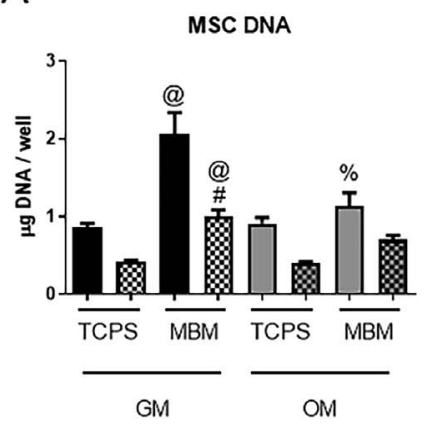

E

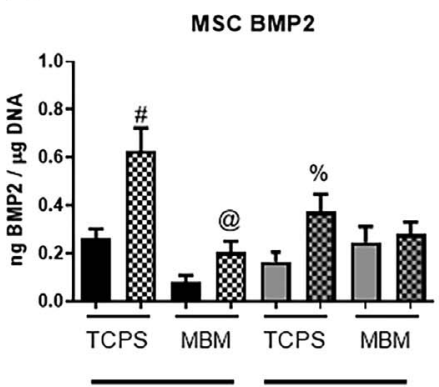

GM
B

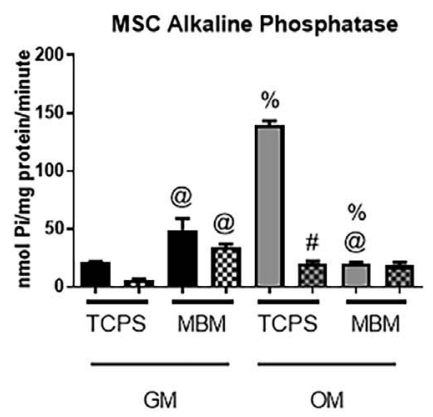

F

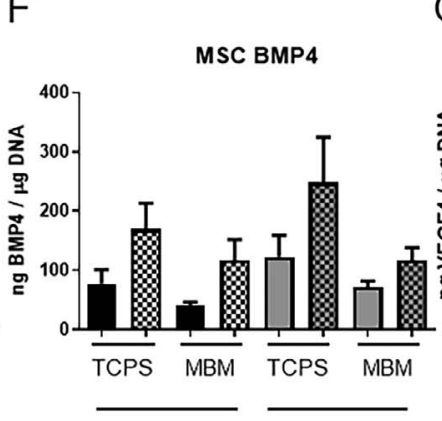

GM
C

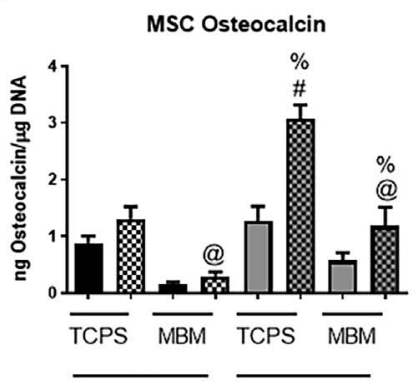

GM

G

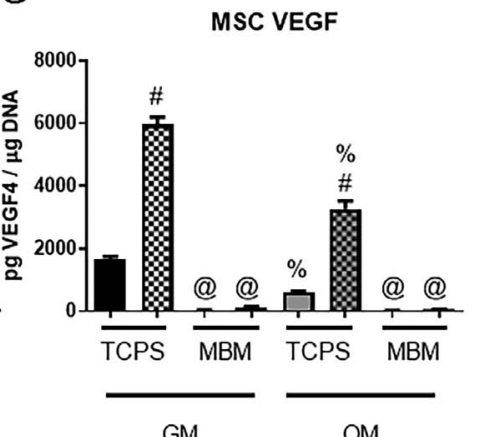

D
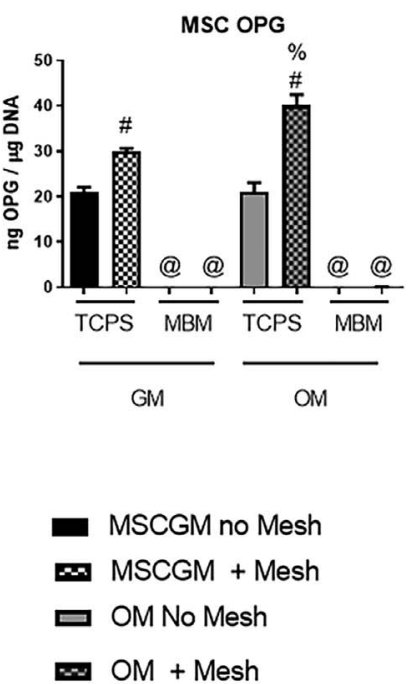

Figure 3. In vitro bone marrow stromal cell (MSC) response of cells cultured on tissue culture plastic (TCPS) or mineralized bone matrix (MBM), with MSC growth media (GM) or osteogenic media (OM), in the presence or absence of polyethylene terephthalate mesh (PET). Response was examined by measuring markers that indicate the initiation of differentiation including (A) DNA; (B) alkaline phosphate specific activity; factors that indicate late stage osteoblast differentiation including (C) osteocalcin and (D) osteoprotegerin (OPG); and production of factors associated with osteogenesis, including bone morphogenetic proteins (BMPs) 2 (E) and 4 (F) and $(\mathrm{G})$ vascular endothelial growth factor-A (VEGF). $P<.05$ \# versus no mesh, @ versus no MBM. \% versus GM.

OPG production was stimulated by the presence of mesh when cells were cultured on TCPS, but when cells were cultured on bone particles, no OPG was detected whether the mesh was present or not (Figure 2d). Cells cultured on particulate made less BMP2 (Figure 2e), BMP4 (Figure 2f), and VEGF (Figure $2 \mathrm{~g}$ ) than cells on TCPS, particularly when cells were cultured on TCPS where mesh was present.

\section{Response of MSCs}

When MSCs were cultured in MSCGM, DNA content was highest on bone particles, whether mesh was present or not (Figure 3a), but this difference was not evident in MSCs cultured in OM. Alkaline phosphatase activity was higher in cultures grown in GM on particulate whether mesh was present or not (Figure 3b). However, when cells were cultured on TCPS in OM, there was a marked increase in alkaline phosphatase activity, which was reduced to control levels by the mesh and in cultures grown on bone particles. Osteocalcin was highest in MSCs cultured on TCPS in OM in the presence of mesh (Figure 3c). Similarly, osteocalcin was higher in cultures on bone particles plus mesh than on bone particles alone. OPG content in cultures grown on TCPS in GM or OM was higher when mesh was present (Figure 3d). No OPG was in media from any cultures grown on bone particles. BMP2 production was greater in cultures grown in GM on both TCPS and particulate when mesh was present (Figure 3e), but only in cultures grown in OM on TCPS with mesh. Substrate, media, and mesh all had no effect on BMP4 production (Figure 3f). VEGF production in cultures grown on TCPS was higher when mesh was present under both media conditions. No VEGF was detected in cultures grown on the bone particles (Figure $3 \mathrm{~g}$ ).

\section{In Vivo Analysis}

\section{Histopathology}

There was no evidence of foreign body/inflammatory reaction or significant pathological changes in any sites treated with PET mesh plus graft. There was no evidence of bone within the interbody space with discectomy alone (Figure 4). Pedicle screw fixation resulted in the most significant amount of bridging trabecular bone through the PET mesh. In sites treated with autograft or allograft/DBM 
Discectomy
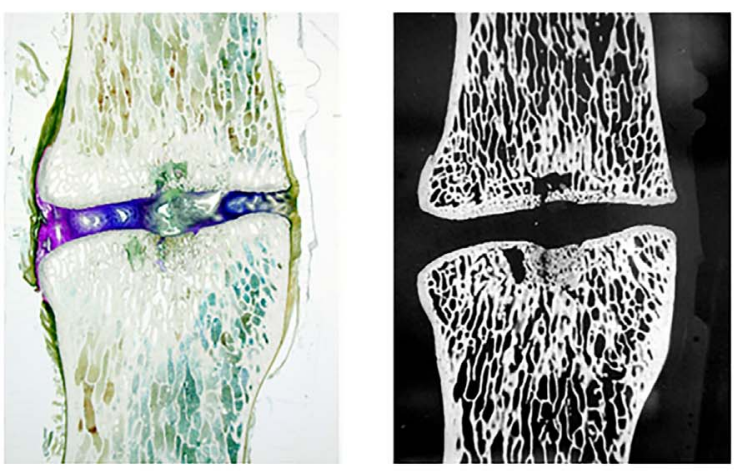

Allograft/DBM

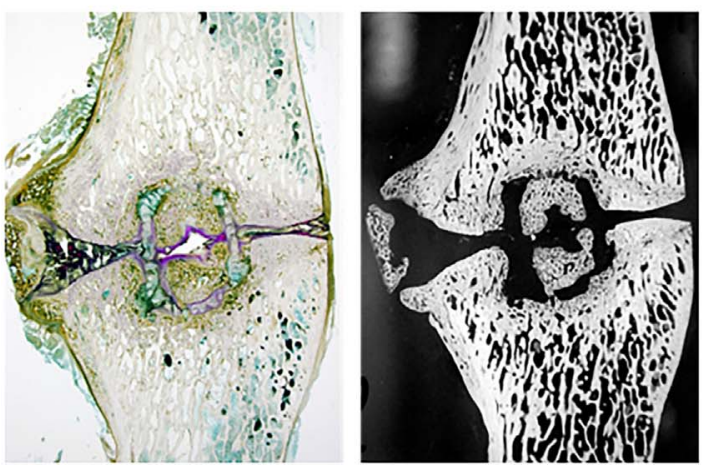

Autograft

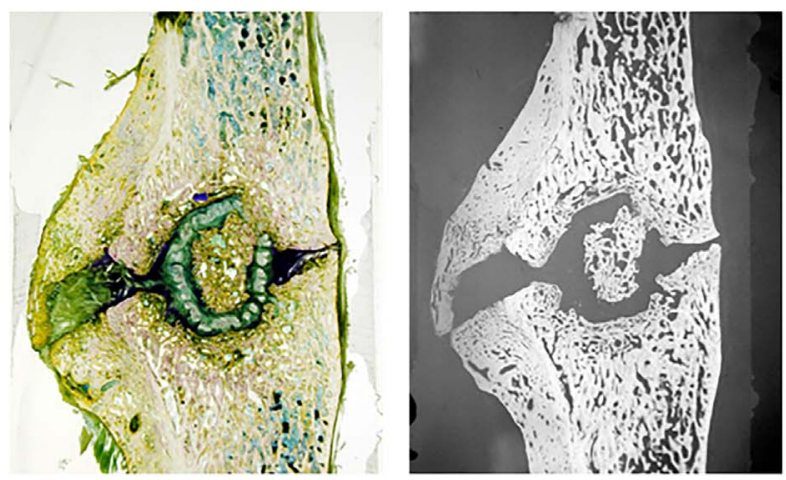

Allo./DBM + Screws

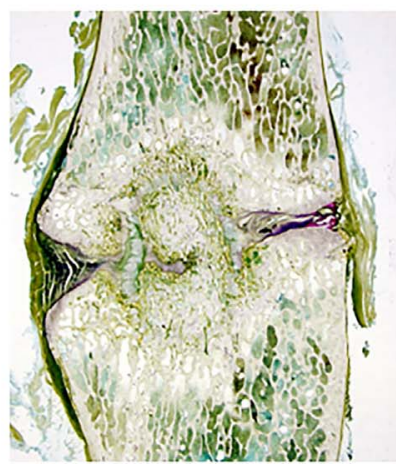

Figure 4. Representative parasagittal images of histologic (left) and microradiograph (right) samples from each interbody treatment including discectomy, autograft, allograft/demineralized bone matrix (DBM), and allograft/DBM plus pedicle screws. One hundred micrograms undecalcified samples were stained using the Villanueva Osteochrome Bone Stain, and embedded in polymethylmethacrylate. Microradiographs of the histologic sections were obtained using a Faxitron x-ray.

without instrumentation, there were interposing pockets of cartilaginous/collagenous tissue, which was actively remodeling. In almost all cases, the trabecular bone within the device was dense woven and sclerotic in most regions, containing normal osteocyte distribution and count and osteoid seam widths. Overall, there appeared to be no signs of osseous pathology due to the PET device or fill materials and all specimens could be characterized as unremarkable and undergoing a normal healing process. These results are summarized in Table 4 and are described in detail.

Discectomy Alone. In most cases, the disc center region appeared to have been disturbed as indicated by disrupted nucleus pulposus and presence of woven bone and cavities along the vertebral endplates. In all cases, a small fibrous nonunion was indicated, containing thick type-I collagenous attachments and cartilaginous remnants. Osteoid seam widths were within normal limits and woven bone within the discectomy regions appeared healthy, without evidence of bone necrosis, inflammatory/giant cell reaction, or other significant histopathology. All specimens demonstrated incomplete trabeculation spanning the disc space and could be histologically characterized as nonunions.

Autograft. In almost all cases, the inner confines of the device were devoid of trabecular bone. Osteo-

Table 4. Analysis of the histologic and microradiographic specimens. ${ }^{*}$ Discectomy is based on an $n=5$; Autograft is based on an $n=4$.

\begin{tabular}{lcccc}
\hline Treatment Group & Fused & Partial & Non-Union & Extra-Articular Bone \\
\hline Discectomy & 0 & 0 & 5 & 1 \\
Autograft & 0 & 1 & 3 & 0 \\
Allograft/DBM & 0 & 4 & 2 & 2 \\
Allograft/DBM screws & 4 & 2 & 0 & 3 \\
\hline
\end{tabular}

Abbreviation: DBM, demineralized bone matrix 


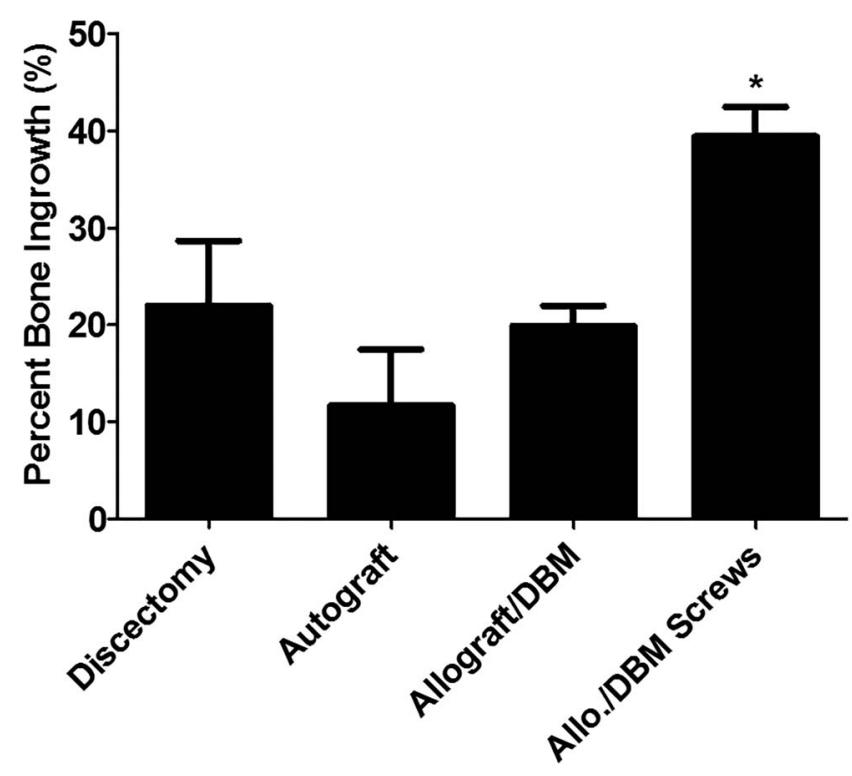

Figure 5. Quantification of bone ingrowth in the microradiographs. ${ }^{*} P<.05$, versus all other groups.

cyte distribution and count were above average within the host and graft regions for all specimens. In many regions directly surrounding the implant site, cartilaginous remnants were undergoing cartilaginous metaplasia. Collagen strands were observed penetrating mesh fenestrations in some areas and osteoid seam widths were within normal limits. Mesh fiber orientation was well preserved, however, in many cases was devoid of fenestrations for trabecular through-growth. Overall, these specimens appeared healthy without evidence of bone necrosis, inflammatory/giant cell reaction, or other significant histopathology.

Allograft/DBM. As a group, these specimens demonstrated more trabecular bone within the device than other groups. There were 3 cases of extra-articular bone, and in these cases, trabecular regions within the device appeared to contain more lamellar than woven bone with normal osteocyte distribution and count and osteoid seam widths, suggesting that the extraarticular bone facilitated maturation of the trabeculae within the device. Pockets of unmineralized type I collagen observed in the middle of the arthrodesis site appeared to have fibers extending away from the unmineralized osteoid bone within the region. As with other treatments, there was no evidence of foreign body reaction, inflammatory reaction, or significant pathological changes observed in these specimens.

Allograft/DBM+Pedicle Screws. Of all the treatments evaluated, these specimens clearly demon- strated the most significant amount of bridging trabecular bone within the confines of the mesh. Two specimens contained an interposing pocket of cartilaginous/collagenous tissue, which was actively remodeling. Mesh fenestrations were well preserved as demonstrated by multiple regions (pathways) of osseointegration. Screw paths were composed of sclerotic bone and without foreign body reaction or significant pathology. Trabecular bone within the device was woven in most regions, containing normal osteocyte distribution and count and osteoid seam widths. There was no evidence of foreign body reaction, inflammatory reaction, or significant pathology observed in these specimens.

\section{Microradiographic Assessment}

Quantification of bone in the microradiographs demonstrated that significant bone ingrowth was achieved only when allograft plus DBM was used and the sites were stabilized by pedicle screws (Figure 5).

\section{Biomechanical Testing}

There were no statistically significant differences in the peak ROM between any of the groups in axial rotation (Figure 6a). Three groups (autograft, allograft/DBM+pedicle screws, and allograft/DBM with pedicle screws removed) had significantly less flexion-extension compared with allograft/DBM alone $(P<.05)$. There was no statistical difference between discectomy and allograft/DBM (Figure $6 \mathrm{~b}$ ). In lateral bending, the allograft/DBM+pedicle screws group had significantly less lateral bending compared with allograft/DBM group. There were no other differences between groups (Figure 6C).

\section{DISCUSSION}

Our results indicate that growth on bone particles stimulates early differentiation of NHOst cells compared with TCPS, but delays terminal differentiation, potentially due to lower production of BMP2 and BMP4. Numerous studies show that cancellous bone chips provide an osteoconductive surface for osteoprogenitor cell migration and proliferation, ${ }^{23,24}$ but terminal differentiation does not occur until proliferation ceases. ${ }^{25,26}$ In addition, progenitor cells resident in the bone chips are known to migrate out and proliferate in culture, ${ }^{27,28}$ contributing to the higher DNA content and lower osteocalcin content of the conditioned media. 
A

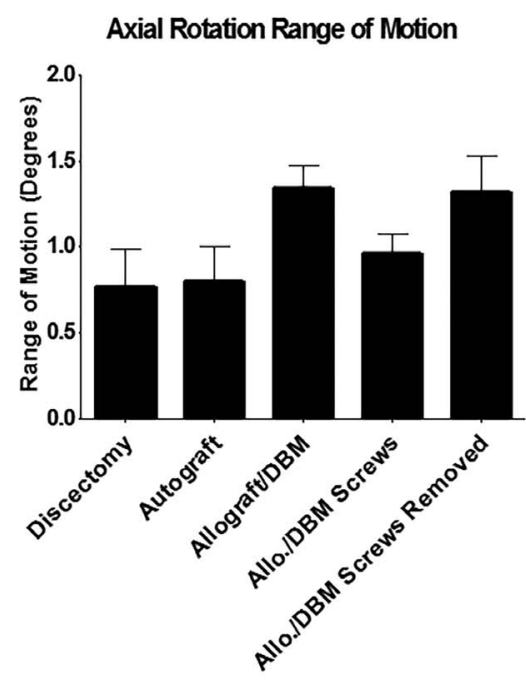

B

Flexion - Extension Range of Motion

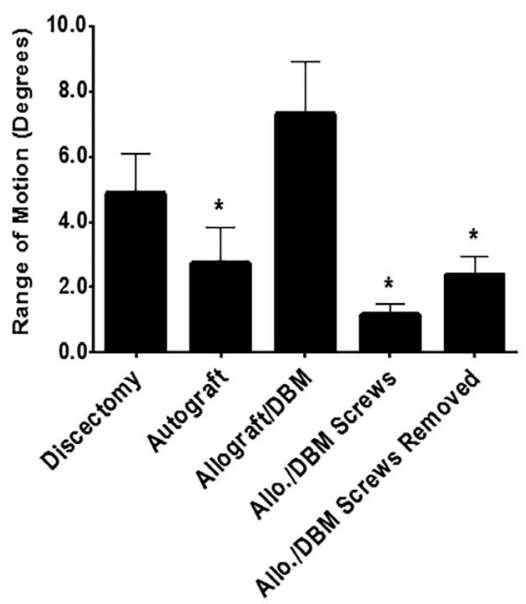

Lateral Bending Range of Motion
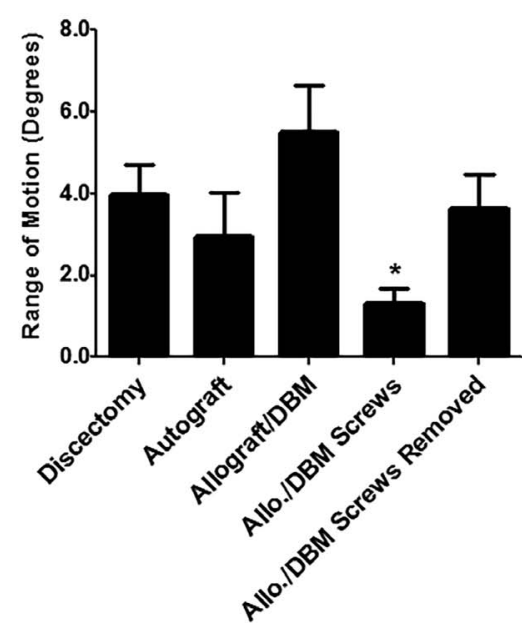

Figure 6. Mechanical testing: (A) axial rotation range of motion; (B) flexion-extension range of motion; and (C) lateral bending range of motion. Sites treated with allograft/demineralized bone matrix (DBM) plus pedicle screws were tested with the screws in place (Allo/DBM Screws) and after removal of the screws with dissection of the facet joints (Allo/DBM Screws Removed). ${ }^{*} P<.05$, versus allograft/DBM alone.

MSCs responded to the bone particles with an increase in DNA compared with cells on TCPS, reflecting the stimulatory effect of the osteoconductive surface on proliferation. MSCs present in the bone chips may also have contributed to the higher DNA content. However, when MSCs were cultured in OM, which stimulates their transition to an osteoblast phenotype, an increase in DNA was not observed. The presence of the mesh reduced DNA content on both surfaces and in both media.

Alkaline phosphatase activity was higher in MSCs cultured in growth media on bone particles and this was not affected by PET mesh, supporting the osteogenic properties of the substrate. OM had a marked stimulatory effect on MSC differentiation on TCPS, as reported in the literature, ${ }^{29,30}$ but this was blocked by the presence of PET mesh. Alkaline phosphatase is an early marker of osteoblast differentiation, and can be misleading as a sole indicator of osteoblast differentiation. Our results show that the stimulatory effect of OM on osteoblast differentiation was evident based on the marked increase in osteocalcin production, a late stage phenotypic marker, when the cells were cultured on TCPS in the presence of the mesh and to a lesser extent on the bone particles. The presence of mesh also caused an increase in production of OPG in cultures grown on TCPS, whether the medium used was GM or OM. Surprisingly, cells cultured on the bone particles did not produce OPG at all, supporting the hypothesis that they were still at a less differentiated state.

The presence of PET mesh caused an increase in BMP2 production when MSCs were cultured on TCPS in both media and in MSCs grown on bone particles in GM, suggesting that part of the stimulatory effect of the mesh was via autocrine/ paracrine action of this osteoinductive factor. ${ }^{31,32}$ Results concerning the potential contribution of BMP4 were less conclusive. However, the data strongly support a role for VEGF-A in mediating the PET mesh effect in cultures grown on TCPS. VEGF-A has been shown to stimulate osteoblast differentiation in a number of studies, ${ }^{33-36}$ but why the presence of PET would cause an increase in this angiogenic factor is not clear, particularly because MSCs grown on bone chips do not produce VEGFA in any detectable amount.

Taken together these results indicate that the mesh does not have a negative impact on cell attachment to bone particles, nor does it impede their ability to proliferate or differentiate along an osteoblastic pathway. The results suggest that the mesh may serve to adsorb factors present in the media that are osteogenic, such that cells migrating through the mesh to the TCPS surface are stimulated to produce factors that stimulate osteoblast differentiation. This property was not evident when NHOst cells or MSCs were grown on an osteoconductive substrate. 
Our in vivo results support the in vitro data, demonstrating that new bone formation was not prevented by the presence of the PET mesh graft containment device. Fusion was enhanced by incorporation of allograft/DBM into the mesh compared with autograft, and this was further enhanced when the device was stabilized using pedicle screws. Bone tethers could be seen extending from the vertebral endplates through the mesh into the packed graft, particularly when the vertebrae were stabilized with screws. Measurement of bone ingrowth on the microradiographs confirmed this impression. Only those sites treated with allograft/ DBM and stabilized with pedicle screws exhibited greater percent bone ingrowth compared with either discectomy or autograft.

Addition of pedicle screw fixation to the allograft/DBM sites resulted in a significant increase in stability in flexion-extension and in lateral bending, likely due to the higher percent of bone ingrowth traversing the vertebral endplate into the PET mesh, resulting in better anchoring of the PET mesh graft construct at the vertebral endplate interface. The percent of bone ingrowth for the allograft/DBM with pedicle screws was approximately twice that of the allograft/DBM group due to the added rigidity across the fusion site from the posterior fixation. A greater extent of bone ingrowth into the mesh due to the added posterior rigidity would augment the load sharing between the screw fixation and PET mesh graft construct across the fusion site. This was indirectly reflected biomechanically by the significant reduction in the ROM measured in flexionextension and lateral bending for the allograft/ $\mathrm{DBM}+$ pedicle screws group. Further evidence that greater bone ingrowth and hence biomechanical stability was not solely related to the added pedicle screw fixation but also to the added stability of the anterior column at the fusion segment was provided once the pedicle screws were removed and the facets were dissected off of the samples and biomechanically retested. Removal of the screws and dissection of the facet joints would theoretically lessen the rigidity and add instability across the fusion site. However, the findings demonstrated a reduction in the ROM evident in flexion-extension and lateral bending for this group, implying that the greater bone ingrowth was responsible for the added biomechanical stability and allowed for improved load sharing across the fusion site.

\section{CONCLUSION}

Taken together these results indicate that PET mesh does not have a negative impact on the ability of cells to attach to bone particles, nor does it impede their ability to proliferate or undergo initial stages of osteoblast differentiation. The mesh stimulates differentiation in cultures grown on TCPS, but it does not impact their differentiation on bone particles in vitro. The data show for the first time that terminal osteoblast differentiation induced by $\mathrm{OM}$ is reduced in cells cultured on bone particles compared with cells grown on conventional TCPS. This may be due to enhanced proliferation. It may also reflect contributions of cells that were resident in the bone particles, as fresh graft was used in this study without prior freezing. This raises questions about the potential to reach conclusions about technologies tested using TCPS that are intended for use under very different clinical conditions. The results clearly demonstrated that bone growth occurs from the vertebral endplates into the graft material within the PET mesh graft containment device. This process was enhanced by additional stabilization with pedicle screws leading to greater bone ingrowth into the PET mesh graft and increased biomechanical stability across the fusion site.

\section{ACKNOWLEDGMENTS}

The authors dedicate this article to the memory of Karen M. Roche, Vice President for Operations and Technology, Spineology, Inc. Karen began her career with Spineology together with its founder, Dr Stephen Kuslich, and was instrumental in the design and development of their OptiMesh technology, which has the goal of promoting biologically based interbody fusions. For many of us, Karen was the heart and soul of the company. She will be missed.

All studies were conducted with approval by the Thomas D. Morris, Inc, Institutional Animal Care and Use Committee.

This work was supported by PHS Grants AR052102 and AR072500, as well as a gift from Spineology, Inc. PET mesh inserts were provided by Spineology. Sheep surgeries, mechanical testing, and histological processing were performed at Thomas D. Morris, Inc (Reisterstown, MD) under the direction of Dr Bryan Cunningham. 


\section{REFERENCES}

1. Chamberlain G, Fox J, Ashton B, Middleton J. Concise review: mesenchymal stem cells: their phenotype, differentiation capacity, immunological features, and potential for homing. Stem Cells. 2007;25(11):2739-2749. doi:10.1634/stemcells.20070197

2. O'Keefe RJ. Cellular and molecular targets to enhance fracture healing and bone formation in aging. Bone. 2010;47:s355-s356. doi:10.1016/j.bone.2010.09.050

3. McAfee PC. Interbody fusion cages in reconstructive operations on the spine. J Bone Joint Surg Am. 1999;81(6):859880. doi:10.2106/00004623-199906000-00014

4. Enders JJ, Coughlin D, Mroz TE, Vira S. Surface technologies in spinal fusion. Neurosurg Clin $N \mathrm{Am}$. 2020;31(1):57-64. doi:10.1016/j.nec.2019.08.007

5. Zhang J-dong, Poffyn B, Sys G, Uyttendaele D. Are stand-alone cages sufficient for anterior lumbar interbody fusion? Orthop Surg. 2012;4(1):11-14. doi:10.1111/j.1757-7861. 2011.00164.x

6. Zdeblick TA, Phillips FM. Interbody cage devices. Spine (Phila Pa 1976). 2003;28(15):S2-S7. doi:10.1097/01. brs.0000076841.93570.78

7. Jain S, Eltorai AEM, Ruttiman R, Daniels AH. Advances in spinal interbody cages. Orthop Surg. 2016;8(3):278-284. doi:10.1111/os.12264

8. Hawasli AH, Khalifeh JM, Chatrath A, Yarbrough CK, Ray WZ. Minimally invasive transforaminal lumbar interbody fusion with expandable versus static interbody devices: radiographic assessment of sagittal segmental and pelvic parameters. Neurosurg Focus. 2017;43(2):E10. doi:10.3171/ 2017.5.FOCUS17197

9. Boyan BD, Ranly DM, Schwartz Z. Use of growth factors to modify osteoinductivity of demineralized bone allografts: lessons for tissue engineering of bone. Dent Clin North Am. 2006;50(2):217-228. doi:10.1016/j.cden.2005.11.007

10. Olivares-Navarrete R, Gittens RA, Schneider JM, et al. Osteoblasts exhibit a more differentiated phenotype and increased bone morphogenetic protein production on titanium alloy substrates than on poly-ether-ether-ketone. Spine $J$. 2012;12(3):265-272. doi:10.1016/j.spinee.2012.02.002

11. Ma JL, Huang F, Wang Y, Luo JY, Deng ZL. Evaluation on effects of bone morphoge netic protein2-9 mediated by coexpressing recombinant adenovirus combined with nano-hydroxyapatite/polyamide 66 cage on lumbar interbody fusion of goats. J Jilin Univ Med Ed. 2012;38(6):1086-1090.

12. Mummaneni PV, Dhall SS, Eck JC, et al. Guideline update for the performance of fusion procedures for degenerative disease of the lumbar spine. Part 11: interbody techniques for lumbar fusion. J Neurosurg Spine. 2014;21(1):67-74. doi:10. 3171/2014.4.SPINE14276

13. Munting E, Faundez A, Manche E. Vertebral reconstruction with cortical allograft: long-term evaluation. Eur Spine J. 2001;10(suppl 2):S153-S157. doi:10.1007/s005860100272

14. Boyan BD, Ranly DM, McMillan J, Sunwoo M, Roche K, Schwartz Z. Osteoinductive ability of human allograft formulations. J Periodontol. 2006;77(9):1555-1563. doi:10.1902/ jop.2006.060019

15. Schultz KD. Chapter 43 - Biologic treatment of osteoporotic compression fractures: OptiMesh. In: Yue JJ, Guyer RD, Johnson JP, Khoo LT, Hochschuler SH, eds. The
Comprehensive Treatment of the Aging Spine. Philadelphia, PA: W.B. Saunders; 2011:265-268. doi:https://doi.org/10.1016/ B978-1-4377-0373-3.10043-0

16. Lam S, Khoo LT. A novel percutaneous system for bone graft delivery and containment for elevation and stabilization of vertebral compression fractures. Technical note. Neurosurg Focus. 2005;18(3):e10. doi:10.3171/foc.2005.18.3.11

17. Boyan BD, Bonewald LF, Paschalis EP, et al. Osteoblast-mediated mineral deposition in culture is dependent on surface microtopography. Calcif Tissue Int. 2002;77(6):519529. doi:10.1007/s00223-001-1114-y

18. Bonewald LF, Harris SE, Rosser J, et al. Von Kossa staining alone is not sufficient to confirm that mineralization in vitro represents bone formation. Calcified Tissue Int. 2003;72:537-547. doi:10.1007/s00223-002-1057-y

19. Gittens RA, Olivares-Navarrete R, Schwartz Z, Boyan BD. Implant osseointegration and the role of microroughness and nanostructures: lessons for spine implants. Acta Biomater. 2014;10(8):3363-3371. doi:10.1016/j.actbio.2014.03.037

20. Magin $\mathrm{MN}$, Delling G. Improved lumbar vertebral interbody fusion using rhOP-1: a comparison of autogenous bone graft, bovine hydroxylapatite (Bio-Oss), and BMP-7 (rhOP-1) in sheep. Spine (Phila Pa 1976). 2001;26(5):469-478. doi:10.1097/00007632-200103010-00009

21. Assad M, Jarzem P, Leroux MA, et al. Porous titaniumnickel for intervertebral fusion in a sheep model: part 1 . histomorphometric and radiological analysis. J Biomed Mater Res - Part B Appl Biomater. 2003;64(2):107-120. doi: 10.1002/ jbm.b. 10530

22. Sandhu HS, Toth JM, Diwan AD, et al. Histologic evaluation of the efficacy of rhBMP-2 compared with autograft bone in sheep spinal anterior interbody fusion. Spine (Phila Pa 1976). 2002;27(6):567-575. doi:10.1097/00007632-20020315000003

23. Brandoff JF, Silber JS, Vaccaro AR. Contemporary alternatives to synthetic bone grafts for spine surgery. Am J Orthop (Belle Mead NJ). 2008;37(8):410-414.

24. Bauer TW, Muschler GF. Bone graft materials: an overview of the basic science. Clin Orthop Rel Res. 2000;(371):10-27. doi:10.1097/00003086-200002000-00003

25. Lian JB, Stein GS. Development of the osteoblast phenotype: molecular mechanisms mediating osteoblast growth and differentiation. Iowa Orthop J. 1995;15:118-140.

26. Lian JB, Stein GS. Chapter 14: The cells of bone. In: Seibel M, Robins S, Bilezikian J, eds. Dynamics of Bone and Cartilage Metabolism. 2nd ed. Cambridge, MA: Academic Press; 2006:221-258. doi:10.1016/B978-012088562-6/50015-7

27. Boskey AL, Robey PG. Chapter 11:The regulatory role of matrix proteins in mineralization of bone. In: Marcus R, Feldman D, Dempster DW, Luckey M, Cauley, eds. Osteoporosis. 4th ed. Cambridge, MA: Academic Press; 2013:235-255. doi:https://doi.org/10.1016/B978-0-12-415853-5.00011-X

28. Robey PG, Termine JD. Human bone cells in vitro. Calcif Tissue Int. 1985;37(5):453-460. doi:10.1007/BF02557826

29. Schwartz Z, Simon BJ, Duran MA, Barabino G, Chaudhri R, Boyan BD. Pulsed electromagnetic fields enhance BMP-2 dependent osteoblastic differentiation of human mesenchymal stem cells. J Orthop Res. 2008;26(9):1250-1255. doi:10.1002/jor.20591

30. Vater C, Kasten P, Stiehler M. Culture media for the 
differentiation of mesenchymal stromal cells. Acta Biomater. 2011;7(2):463-477. doi:10.1016/j.actbio.2010.07.037

31. Liu H, Xu GW, Wang YF, et al. Composite scaffolds of nano-hydroxyapatite and silk fibroin enhance mesenchymal stem cell-based bone regeneration via the interleukin 1 alpha autocrine/paracrine signaling loop. Biomaterials. 2015;49:103112. doi:10.1016/j.biomaterials.2015.01.017

32. Xiong L, Zeng J, Yao A, et al. BMP2-loaded hollow hydroxyapatite microspheres exhibit enhanced osteoinduction and osteogenicity in large bone defects. Int $J$ Nanomedicine. 2015;10:517-526. doi:10.2147/IJN.S74677

33. Liu Y, Berendsen AD, Jia S, et al. Intracellular VEGF regulates the balance between osteoblast and adipocyte differentiation. J Clin Invest. 2012;122(9):3101-3113. doi:10. 1172/JCI61209

34. $\mathrm{Hu} \mathrm{K}$, Olsen BR. Osteoblast-derived VEGF regulates osteoblast differentiation and bone formation during bone repair. J Clin Invest. 2016;126(2):509-526. doi:10.1172/JCI82585

35. Deckers MML, Van Bezooijen RL, Van Geertje Horst DER, et al. Bone morphogenetic proteins stimulate angiogenesis through osteoblast-derived vascular endothelial growth factor A. Endocrinology. 2002;143(4):1545-1553. doi:10.1210/ endo.143.4.8719

36. Berendsen AD, Olsen BR. How vascular endothelial growth factor-A (VEGF) regulates differentiation of mesenchymal stem cells. J Histochem Cytochem. 2014;62(2):103-108. doi: $10.1369 / 0022155413516347$

Disclosures and COl: B.D.B., L.F., and M.B.S. are consultants for Spineology. The other authors received no funding for this study and report no conflicts of interest.

Corresponding Author: Barbara D. Boyan, $\mathrm{PhD}$, College of Engineering, Virginia Commonwealth University, 601 West Main St, Richmond, VA 23284. Phone: 1-804-828-0190; Email: bboyan@ vcu.edu.

Published 9 December 2020

This manuscript is generously published free of charge by ISASS, the International Society for the Advancement of Spine Surgery. Copyright (C) 2020 ISASS. To see more or order reprints or permissions, see http://ijssurgery.com. 